\title{
Environment effect on cross-polarized excitons in carbon nanotubes
}

\author{
Seiji Uryu ${ }^{1}$ and Tsuneya Ando $^{2}$ \\ ${ }^{1}$ Department of Materials Science and Engineering, Iwate University 4-3-5 Ueda, Morioka, Iwate 020-8551, Japan \\ ${ }^{2}$ Department of Physics, Tokyo Institute of Technology 2-12-1 Ookayama, Meguro-ku, Tokyo 152-8551, Japan
}

(Received 17 April 2012; revised manuscript received 27 July 2012; published 7 September 2012)

\begin{abstract}
Effects of environmental dielectric materials on cross-polarized excitons of semiconducting carbon nanotubes are theoretically studied in an effective-mass scheme. When a nanotube is surrounded by a medium, the bright exciton, shifted to the high-energy side by depolarization effect, becomes weaker and disappears with the increase of the dielectric constant. When a nanotube contains a medium inside, the bright exciton becomes more eminent. The dark exciton, brightened due to electron-hole asymmetry, is enhanced with the increase of the dielectric constant both for outside and inside materials. Consequently, the relative intensity of the brightened dark exciton normalized by the bright exciton is strongly enhanced by environmental screening, in particular, for outside materials.
\end{abstract}

DOI: 10.1103/PhysRevB.86.125412

PACS number(s): 78.67.Ch, 73.22.Lp

\section{INTRODUCTION}

Because a carbon nanotube is full of surface, electrons in a nanotube are strongly subjected to effects of a surrounding environment. In fact, energy levels of optically excited excitons were shown to be sensitive to environmental conditions for parallel polarization in which the electric field of light is parallel to the axis, ${ }^{1-16}$ indicating that the Coulomb interaction is effectively modulated by the environments. The purpose of this study is to theoretically clarify dielectric environment effects on cross-polarized excitons in carbon nanotubes.

Effects of the Coulomb interaction are known to play a significant role in carbon nanotubes because of one dimension. ${ }^{17-19}$ In fact, it was theoretically predicted that the band gaps are considerably enhanced due to the Coulomb interaction, but a large part of the enhancement is compensated by the exciton binding energy and the exciton lies slightly above each band edge without interaction effects. ${ }^{20,21}$ This was confirmed by later theoretical studies. ${ }^{22-30}$ Experiments on an excitation energy associated with each band ${ }^{31-34}$ and two-photon absorption ${ }^{35-37}$ proved the significant role of exciton effects in nanotubes for parallel polarization.

For cross polarization, in which the electric field of light is perpendicular to the axis, the spectrum is strongly affected by a depolarization field between plus and minus charges induced by light at opposite sides of the tube cross section. In the presence of this depolarization effect, peaks associated with van Hove singularity in interband continuum are completely suppressed, ${ }^{38,39}$ but excitons remain as sharp peaks in absorption or emission spectra. $26,28,40-44$ Thus observation of absorption peaks for cross polarization is another clear evidence of excitons in nanotubes. ${ }^{41,42,44}$ Further recent studies for cross-polarized excitons revealed that dark excitons, located below bright excitons in energy, become optically allowed due to weak electron-hole asymmetry. ${ }^{4-47}$

Optical spectra of carbon nanotubes have been measured in various environments such as for self-assembled tubes, ${ }^{3,5,48-50}$ tubes suspended in air, ${ }^{4,7,8,10,12,51}$ and tubes in the presence of surrounding materials such as surfactants and solvents. ${ }^{1,3,6,7,9,11,13-16,34}$ It has often been shown that exciton energies in the above environments are reduced in comparison with those in tubes in air or vacuum. In fact, in tubes in solvents, energies decrease by about several tens milli electron volts depending on the dielectric constants. ${ }^{9,15}$ It should be noted that materials intercalated inside nanotubes ${ }^{52,53}$ can also be considered as environment for the nanotubes. As to theoretical studies, in addition to the simplest models using a single screening parameter, ${ }^{22,54-56}$ analytical ${ }^{57,58}$ and phenomenological ${ }^{59-61}$ considerations of configurations of environmental dielectrics have showed that observed exciton energies are reasonably reproduced. Calculations of selfassembled tubes were recently reported. ${ }^{62,63}$

In cross-polarized geometry, dielectric environment significantly modifies the depolarization effect as well as the exciton energy. In this paper, we theoretically clarify such effects in an effective-mass approximation with higher-order corrections. The paper is organized as follows. In Sec. II, a model and method are presented. Numerical results are shown in Sec. III and discussed in Sec. IV. A summary and conclusion are given in Sec. V.

\section{MODEL AND METHOD}

\section{A. Effective-mass approximation}

Carbon nanotubes are rolled up graphene sheets where atoms connected by chiral vector $\mathbf{L}$ defining the tube circumference are overlapped. In a graphene, the conduction and valence bands consisting of $\pi$ states cross at $K$ and $K^{\prime}$ points where the Fermi energy is located.

Electrons near the $K$ point are described by a $\mathbf{k} \cdot \mathbf{p}$ Hamiltonian: ${ }^{64-66}$

$$
\mathcal{H}=\left(\begin{array}{cc}
\sqrt{3} a \gamma S \hat{\mathbf{k}}^{2} / 2 & h(\hat{\mathbf{k}}) \\
h(\hat{\mathbf{k}})^{\dagger} & \sqrt{3} a \gamma S \hat{\mathbf{k}}^{2} / 2
\end{array}\right),
$$

with

$$
h(\hat{\mathbf{k}})=\gamma\left(\hat{k}_{x}-i \hat{k}_{y}\right)+\frac{\beta a \gamma}{4 \sqrt{3}} e^{3 i \eta}\left(\hat{k}_{x}+i \hat{k}_{y}\right)^{2},
$$

where $\gamma$ is a band parameter, $\hat{\mathbf{k}}=\left(\hat{k}_{x}, \hat{k}_{y}\right) \equiv-i \vec{\nabla}$ is a wave vector operator, $a=2.46 \AA$ is the lattice constant, $\eta$ is the chiral angle denoting the direction of $\mathbf{L}$, and $S$ is a dimensionless parameter characterizing electron-hole asymmetry. The parameter $\beta$ denotes the strength of trigonal warping. 
Namely, the equienergy line is perfectly circular for $\beta=0$ and deformed toward an equilateral triangle with the increase of $|\beta|$. In a nearest-neighbor tight-binding model, $\beta=1$. $^{64,67}$

Electronic states for a nanotube with a sufficiently large diameter are obtained by imposing the following boundary condition for a two-component envelope function $\mathbf{F}(\mathbf{r})$ :

$$
\mathbf{F}(\mathbf{r}+\mathbf{L})=\mathbf{F}(\mathbf{r}) \exp \left[2 \pi i\left(-\frac{v}{3}+\varphi\right)\right],
$$

with $v$ being an integer determined as $v=0$ or \pm 1 corresponding to $\mathbf{L}$ and $\varphi$ being effective magnetic flux threading the tube cross section which describes effects of curvature and lattice distortion. The nanotube becomes a metal for $v=0$ and a semiconductor for $v= \pm 1$. The effective flux is given by ${ }^{68-73}$

$$
\varphi=-\frac{2 \pi}{4 \sqrt{3}} \frac{a}{L} p \cos 3 \eta,
$$

where $L=|\mathbf{L}|$ and $p$ is a dimensionless parameter satisfying $-0.5 \lesssim p \lesssim 0$.

The wave function is given by a plane wave, $\mathbf{F}(\mathbf{r}) \propto$ $e^{i \kappa_{\nu}(n, \varphi) x+i k y}$, where $k$ and $\kappa_{v}(n, \varphi)$ are the wave vectors along the axis and circumference, respectively, with

$$
\kappa_{\nu}(n, \varphi)=\frac{2 \pi}{L}\left(n-\frac{v}{3}+\varphi\right) .
$$

For the $K^{\prime}$ point, the Hamiltonian matrix is given by the complex conjugate of $\mathcal{H}$ after replacing $\beta$ with $-\beta$, and the boundary condition is given by Eq. (3) with replacement of $v$ and $\varphi$ by $-v$ and $-\varphi$, respectively.

The band parameter $\gamma$ is related to resonance integral $-\gamma_{0}$ between neighboring $\pi$ orbitals in a tight-binding model through $\gamma=\sqrt{3} \gamma_{0} a / 2$. Previous studies revealed that $\gamma_{0} \approx$ $2.7 \mathrm{eV}(\gamma \approx 5.8 \mathrm{eVA})$ can well reproduce experiments. ${ }^{21,74}$ For the higher-order corrections, it was shown that values around $\beta=1.5$ and $p=-0.2$ reproduce experimental family patterns well. ${ }^{75}$ Thus results for these parameters will be shown when they are considered. As to the electron-hole asymmetry, $S \sim 0.13$ has usually been assumed. ${ }^{76}$ Therefore we use $S=0.1$ or 0.2 when it is taken into account. Actually, results are dependent only on $|S|$ and independent of the sign of $S$.

The exciton wave function is a linear combination of electron-hole pair states. Expansion coefficients are obtained by solving an equation of motion for an electron-hole pair under attractive interaction..$^{20,21,77}$ We shall not consider an electron-hole exchange interaction included in a TammDancoff type approximation for description of depolarization effects. For cross polarization, excitons with momentum $\pm 2 \pi / L$ in the circumference direction are excited. In calculations of interaction effects on the band structure, a screened Hartree-Fock approximation is used and the attractive interaction between an electron and hole is introduced by using the Coulomb interaction screened by a static dielectric function, ${ }^{20,21,77}$ which was shown to be sufficient by comparison with results of calculations in a higher $G W$-type approximation. $^{78,79}$

\section{B. Environment effect}

A dielectric function is written as

$$
\varepsilon_{n}(q, \omega)=1+V_{n}(q)\left[\Pi_{n}^{\prime}(q, \omega)+\Pi_{n}(q, \omega)\right],
$$

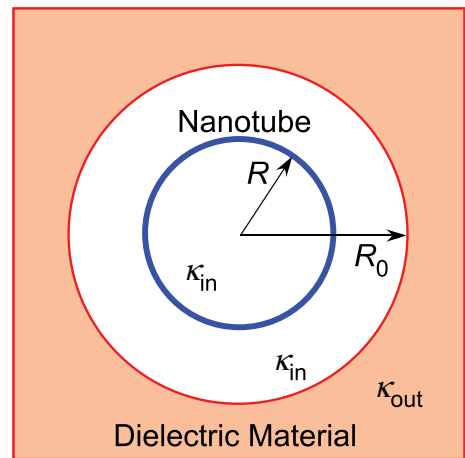

(a)

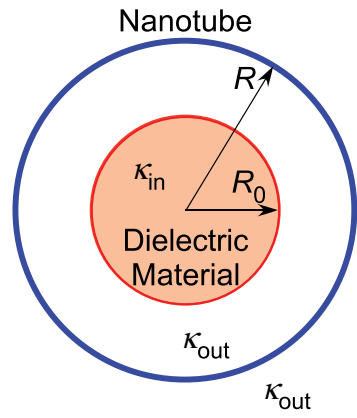

(b)
FIG. 1. (Color online) Schematic illustration of a nanotube with radius $R=L / 2 \pi$ (a) in environmental dielectric material with hollow cylinder with radius $R_{0}$ and (b) containing cylindrical material with radius $R_{0}$.

where integer $n$ characterizes the wave vector in the circumferential direction, $q$ is the wave vector in the tube axis direction, $V_{n}(q)$ is the Fourier transform of the Coulomb interaction, $\Pi_{n}(q, \omega)$ is a polarization function due to electrons near the Fermi energy, and $\Pi_{n}^{\prime}(q, \omega)$ is that due to other electrons in $\sigma$ bands, core states, and the $\pi$ bands away from the $K$ and $K^{\prime}$ points. The polarization function $\Pi_{n}^{\prime}(q, \omega)$ is written using a phenomenological dielectric constant $\kappa$ as ${ }^{57}$

$$
V_{n}^{0}(q) \Pi_{n}^{\prime}(q, \omega) \approx \kappa-1,
$$

where the Fourier transform of the Coulomb interaction in the absence of environment materials is given by

$$
V_{n}^{0}(q)=2 e^{2} F_{n}(q R)
$$

with

$$
F_{n}(q R)=I_{n}(q R) K_{n}(q R),
$$

and $I_{n}(z)$ and $K_{n}(z)$ being modified Bessel functions of the first and the second kind, respectively.

When a medium with a hollow and coaxial cylinder with radius $R_{0}$ and dielectric constant $\kappa_{\text {out }}$ is placed around a nanotube with radius $R$, as shown in Fig. 1(a), $V_{n}(q)$ becomes $^{57}$

$$
V_{n}(q)=\frac{2 e^{2}}{\kappa_{\text {in }}} F_{n}(q R) \frac{1+\delta_{\kappa} G_{n}\left(q R_{0}\right) \xi_{n}\left(q R, q R_{0}\right)}{1+\delta_{\kappa} G_{n}\left(q R_{0}\right)},
$$

where $\kappa_{\text {in }}$ is a background dielectric constant,

$$
\delta_{\kappa}=\frac{\kappa_{\mathrm{out}}}{\kappa_{\mathrm{in}}}-1,
$$

and

$$
\begin{gathered}
G_{n}\left(q R_{0}\right)=-\left.q R_{0} I_{n}\left(q R_{0}\right) \frac{d K_{n}(z)}{d z}\right|_{z=q R_{0}}, \\
\xi_{n}\left(q R, q R_{0}\right)=1-\frac{I_{n}(q R) K_{n}\left(q R_{0}\right)}{K_{n}(q R) I_{n}\left(q R_{0}\right)} .
\end{gathered}
$$

The screened Coulomb interaction becomes

$$
\frac{V_{n}(q)}{\varepsilon_{n}(q, 0)}=\frac{V_{n}^{0}(q)}{\kappa \tilde{\varepsilon}_{n}(q)},
$$


where

$$
\tilde{\varepsilon}_{n}(q)=\tilde{\varepsilon}_{n}^{0}(q)+\frac{1}{\kappa}\left[\kappa_{\mathrm{in}} \frac{1+\delta_{\kappa} G_{n}\left(q R_{0}\right)}{1+\delta_{\kappa} G_{n}\left(q R_{0}\right) \xi_{n}\left(q R, q R_{0}\right)}-1\right],
$$

with $\tilde{\varepsilon}_{n}^{0}(q)$ being the dielectric function for a nanotube in the absence of surrounding material:

$$
\tilde{\varepsilon}_{n}^{0}(q)=1+\frac{V_{n}^{0}(q)}{\kappa} \Pi_{n}(q, 0) .
$$

This shows that effects of environmental dielectric materials appear only in the form of the added dielectric constant given by the second term in the right hand side of Eq. (15), although being strongly dependent on $q$ and $R_{0} / R$. It is assumed in the following that the background is vacuum, i.e., $\kappa_{\text {in }}=1$.

When a coaxial cylindrical material with radius $R_{0}$ and dielectric constant $\kappa_{\text {in }}$ is inserted into a nanotube, as shown in Fig. 1(b), $V_{n}(q)$ is given by ${ }^{57}$

$$
V_{n}(q)=\frac{2 e^{2}}{\kappa_{\mathrm{out}}} F_{n}(q R) \frac{1+\delta_{\kappa}^{\prime} G_{n}^{\prime}\left(q R_{0}\right) \xi_{n}^{\prime}\left(q R, q R_{0}\right)}{1+\delta_{\kappa}^{\prime} G_{n}^{\prime}\left(q R_{0}\right)},
$$

where $\kappa_{\text {out }}$ is the background dielectric constant,

$$
\delta_{\kappa}^{\prime}=\frac{\kappa_{\text {in }}}{\kappa_{\text {out }}}-1,
$$

and

$$
\begin{gathered}
G_{n}^{\prime}\left(q R_{0}\right)=\left.q R_{0} K_{n}\left(q R_{0}\right) \frac{d I_{n}(z)}{d z}\right|_{z=q R_{0}}=1-G_{n}\left(q R_{0}\right) \\
\xi_{n}^{\prime}\left(q R, q R_{0}\right)=1-\frac{I_{n}\left(q R_{0}\right) K_{n}(q R)}{K_{n}\left(q R_{0}\right) I_{n}(q R)} .
\end{gathered}
$$

Then, the dielectric function $\tilde{\varepsilon}_{n}(q)$, which is defined by Eq. (14), becomes

$$
\tilde{\varepsilon}_{n}(q)=\tilde{\varepsilon}_{n}^{0}(q)+\frac{1}{\kappa}\left[\kappa_{\mathrm{out}} \frac{1+\delta_{\kappa}^{\prime} G_{n}^{\prime}\left(q R_{0}\right)}{1+\delta_{\kappa}^{\prime} G_{n}^{\prime}\left(q R_{0}\right) \xi_{n}^{\prime}\left(q R, q R_{0}\right)}-1\right] .
$$

Effects of environmental dielectric materials again appear only in the form of the added dielectric constant. For the inside materials, the background is again set to be vacuum, i.e., $\kappa_{\text {out }}=$ 1 , in the following.

Characteristic features of screening due to the outside and inside materials can be most clearly seen in the case of $R_{0}=R$. Then, we have $\xi_{n}(q R, q R)=\xi_{n}^{\prime}(q R, q R)=0$. Thus the dielectric functions for outside and inside materials are, respectively, given by

$$
\begin{aligned}
& \tilde{\varepsilon}_{n}(q)=1+\frac{2 e^{2}}{\kappa} F_{n}(q R) \Pi_{n}(q)+\frac{\kappa_{\mathrm{out}}-1}{\kappa} G_{n}(q R), \\
& \tilde{\varepsilon}_{n}(q)=1+\frac{2 e^{2}}{\kappa} F_{n}(q R) \Pi_{n}(q)+\frac{\kappa_{\mathrm{in}}-1}{\kappa} G_{n}^{\prime}(q R) .
\end{aligned}
$$

In Eq. (22), for sufficiently large $\kappa_{\text {out }}, \tilde{\varepsilon}_{n}(q)$ becomes proportional to $\kappa_{\text {out }}$ for a long wavelength because of $G_{n}(q R) \approx 1$ for $q R \ll 1$ and $n=0$. This leads to drastic reduction of the Coulomb interaction. While in Eq. (23), $\tilde{\varepsilon}_{n}(q)$ in the long-wavelength region remains finite for $\kappa_{\text {in }} \rightarrow \infty$, because of $G_{n}^{\prime}(q R)=1-G_{n}(q R) \approx 0$ for $q R \ll 1$ and $n=0$. As $R_{0}$ deviates from $R$, this big difference is rapidly reduced.

The effective distance between the nanotube and a dielectric material is likely to be dependent on details of the material property, but is expected to be of the order of the interlayer distance in bulk graphite, which is $0.334 \mathrm{~nm}$. For typical nanotubes such as the $(10,10)$ tube, the radius is $R \approx 0.68 \mathrm{~nm}$, giving $R_{0} / R \gtrsim 1.5$ for the outside material and $R / R_{0} \gtrsim 1.5$ for the inside material. With the increase of the tube diameter, these ratios become smaller.

It should be pointed out that the effective dielectric constants $\kappa_{\text {out }}$ and $\kappa_{\text {in }}$ of polar material are likely to be different from static value $\varepsilon_{0}$ but closer to high-frequency value $\varepsilon_{\infty}$. Usually, $\varepsilon_{0}$ contains contributions of lattice vibrations or orientation change of molecules. Because such slow responses have characteristic frequencies much smaller than the electron energy (typically band gaps) and the exciton binding energy, they do not contribute to the renormalization of the electron energy and the exciton binding. We should use the dielectric constant of environmental material roughly corresponding to the band gap and the exciton binding energy, which are not so much different from each other in the present system.

\section{Depolarization effect}

For cross polarization, the depolarization effect has to be considered. Among various theoretical treatments of this effect, ${ }^{38,39,80-83}$ the self-consistent field method is used in the following because of its reliability. ${ }^{84}$ We shall not use a Tamm-Dancoff-type approximation, which is regarded as an approximate version of the self-consistent method, because it can sometimes give quantitatively different results. ${ }^{83,84}$

First, the dynamical conductivity without the depolarization effect, $\sigma_{x x}^{l}(\omega)$, where $l= \pm 1$ indicates the wave vector in the circumference direction, is obtained from the above cross-polarized excitons with the circumferential momentum $2 \pi \hbar l / L$ by using the Kubo formula. ${ }^{85}$ Then, absorption power including the depolarization effect is characterized by the real part of $\bar{\sigma}_{x x}(\omega)$, which is given by

$$
\bar{\sigma}_{x x}(\omega)=\frac{1}{2}\left[\bar{\sigma}_{x x}^{+1}(\omega)+\bar{\sigma}_{x x}^{-1}(\omega)\right],
$$

where

$$
\bar{\sigma}_{x x}^{l}(\omega)=\frac{\sigma_{x x}^{l}(\omega)}{\varepsilon_{x x, l}(\omega)},
$$

and $\varepsilon_{x x, l}(\omega)$ describing the depolarization effect is given similarly to Eq. (6) by

$$
\varepsilon_{x x, l}(\omega)=1+V_{l}(0)\left[\Pi_{l}^{\prime}(0, \omega)+\Pi_{l}(0, \omega)\right] .
$$
have

With the use of the approximation given by Eq. (7), ${ }^{38,39}$ we

$$
\begin{aligned}
\varepsilon_{x x, l}(\omega) & =1+\frac{V_{l}(0)}{V_{l}^{0}(0)}(\kappa-1)+\frac{4 \pi^{2} i|l|}{L \omega} \frac{V_{l}(0)}{V_{l}^{0}(0)} \sigma_{x x}^{l}(\omega) \\
& =\kappa\left(1-p_{l}\right)+\frac{4 \pi^{2} i|l| q_{l}}{L \omega} \sigma_{x x}^{l}(\omega),
\end{aligned}
$$




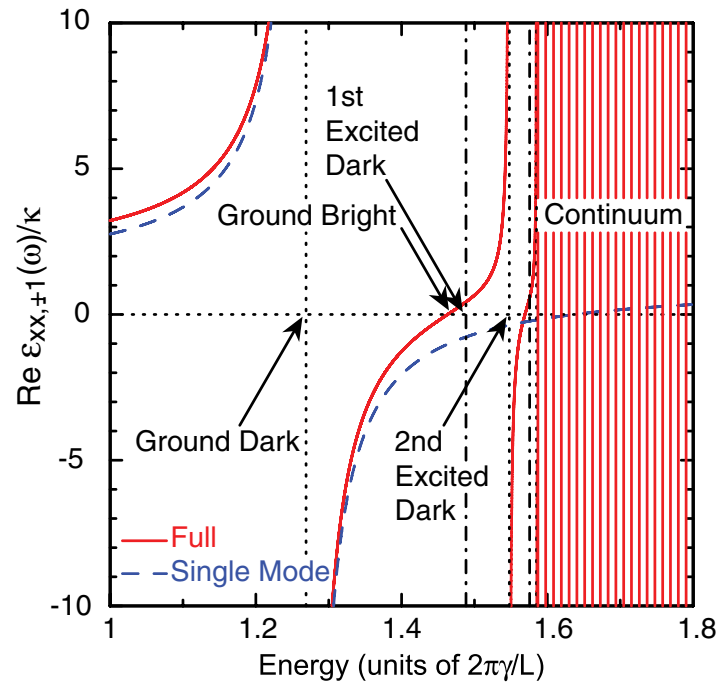

FIG. 2. (Color online) Real part of $\varepsilon_{x x, \pm 1}(\omega)$. Vertical dotted lines denote dark exciton energies and a region with dense vertical lines indicates interband continuum. Dash-dotted lines indicate exciton energies which do not contribute to $\sigma_{x x}^{l}(\omega)$ due to parity symmetry. Energy units are chosen as a typical kinetic energy $2 \pi \gamma / L$.

where $p_{l}$ and $q_{l}$ depend only on $|l|$ and are given for the outside material by

$$
\begin{gathered}
p_{l}=\left(1-\frac{1}{\kappa}\right)\left(1-\frac{\zeta_{l}}{\kappa_{\mathrm{in}}}\right), \\
q_{l}=\frac{\zeta_{l}}{\kappa_{\mathrm{in}}}, \\
\zeta_{l}=1-\frac{\frac{1}{2} \delta_{\kappa}}{1+\frac{1}{2} \delta_{\kappa}}\left(\frac{R}{R_{0}}\right)^{2|l|},
\end{gathered}
$$
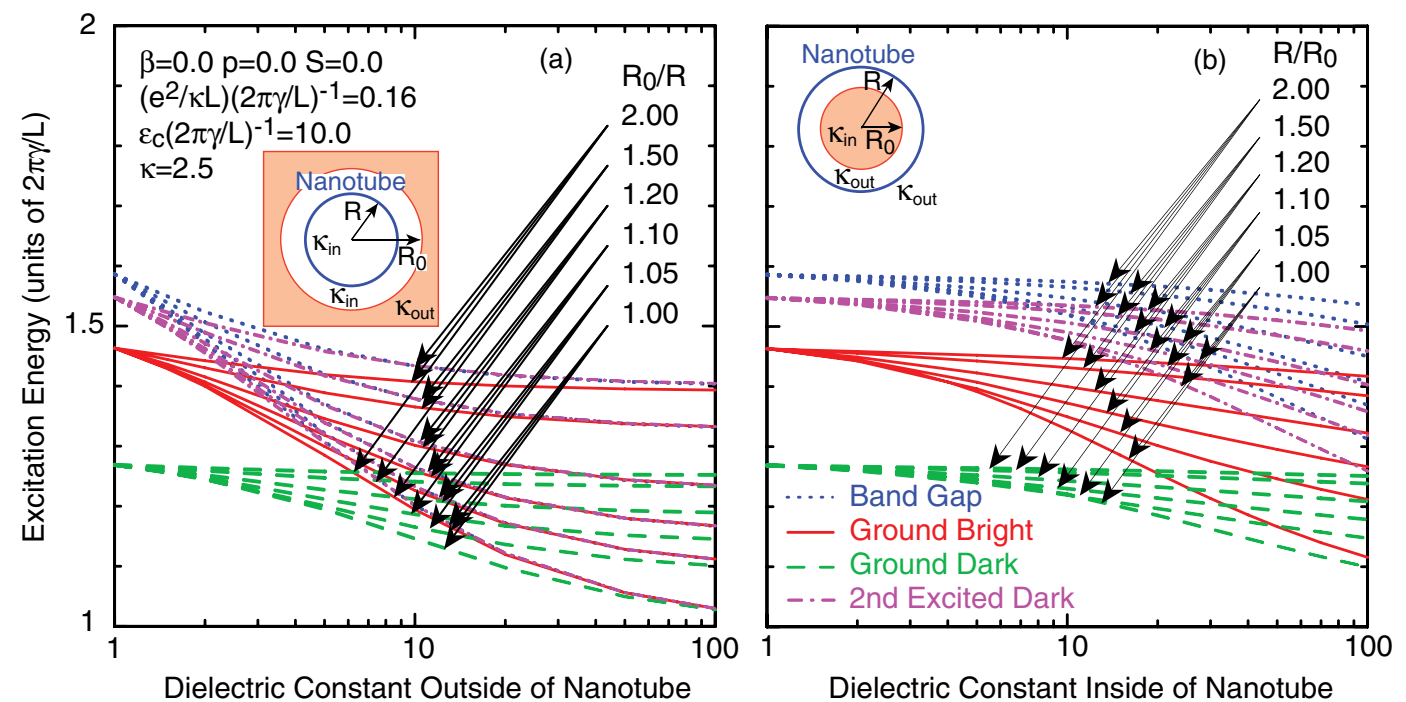

FIG. 3. (Color online) Excitation energy for tubes with (a) outside and (b) inside materials as a function of the dielectric constant in the lowest-order effective-mass approximation, i.e., $\beta=p=S=0$. Solid lines denote those of the lowest bright excitons, dotted lines band gaps, and dashed and dash-dotted lines the lowest and second excited dark excitons, respectively. The ratio between $R$ and $R_{0}$ is $2,1.5,1.2,1.1,1.05$, and 1 from top to bottom. The cutoff energy for the $\pi$ bands is $\varepsilon_{c}(2 \pi \gamma / L)^{-1}=10$.

$$
\begin{gathered}
p_{l}=\left(1-\frac{1}{\kappa}\right)\left(1-\frac{\zeta_{l}^{\prime}}{\kappa_{\mathrm{out}}}\right), \\
q_{l}=\frac{\zeta_{l}^{\prime}}{\kappa_{\mathrm{out}}}, \\
\zeta_{l}^{\prime}=1-\frac{\frac{1}{2} \delta_{\kappa}^{\prime}}{1+\frac{1}{2} \delta_{\kappa}^{\prime}}\left(\frac{R_{0}}{R}\right)^{2|l|},
\end{gathered}
$$

with $\kappa_{\text {out }}=1$. It should be noted that the expressions of $\varepsilon_{x x, l}(\omega)$ for the outside and inside materials are apparently same when $\kappa_{\text {out }}$ and $R_{0} / R$ for the former are replaced by $\kappa_{\text {in }}$ and $R / R_{0}$, respectively. to an applied electric field in an asymptotic external region far away from the tube. The field at the nanotube is also influenced by dielectric environments. In order to see the response of the nanotube itself in the following, therefore, we shall consider conductivity $\tilde{\sigma}_{x x}(\omega)$ for electric field just at the tube surface. This is achieved by defining

$$
\begin{gathered}
\tilde{\varepsilon}_{x x, l}(\omega)=\frac{\varepsilon_{x x, l}(\omega)}{\kappa\left(1-p_{l}\right)}=1+\frac{4 \pi^{2} i|l| q_{l}}{\kappa L \omega\left(1-p_{l}\right)} \sigma_{x x}^{l}(\omega), \\
\tilde{\sigma}_{x x}^{l}(\omega)=\frac{\sigma_{x x}^{l}(\omega)}{\tilde{\varepsilon}_{x x, l}(\omega)} .
\end{gathered}
$$

Obviously, we have

$$
\tilde{\sigma}_{x x}(\omega)=\frac{1}{2}\left[\tilde{\sigma}_{x x}^{+1}(\omega)+\tilde{\sigma}_{x x}^{-1}(\omega)\right]=\kappa\left(1-p_{l}\right) \bar{\sigma}_{x x}(\omega) .
$$

In the absence of environmental screening, this $\tilde{\sigma}_{x x}(\omega)$ reduces to the conductivity used previously. ${ }^{40,47,86}$

\section{Cross-polarized exciton}

Optically allowed excitation energies for cross-polarization are given by zero points of $\varepsilon_{x x, l}(\omega)$ in Eq. (26). ${ }^{40}$
This conductivity $\bar{\sigma}_{x x}(\omega)$ characterizes the optical response 
Figure 2 shows a typical example of the real part of $\varepsilon_{x x, l}(\omega) / \kappa$ for $\kappa_{\text {out }}=\kappa_{\text {in }}=1$ obtained in the lowest-order effective-mass scheme, i.e., $\beta=p=S=0$. This function diverges to plus (minus) infinity on the lower (higher) side of exciton energies without the depolarization effect, i.e., poles of $\sigma_{x x}^{l}(\omega)$, which are denoted by vertical dotted lines. Then, zero points of $\operatorname{Re}\left[\varepsilon_{x x, l}(\omega)\right]$ are located on the higher energy side of the corresponding exciton without the depolarization effect but they are always below the next higher exciton level.

The dashed line in Fig. 2 shows the results when all excitations other than the lowest exciton are neglected. It clearly shows that in this case the energy of the bright exciton is well inside the interband continuum due to the strong depolarization effect. Therefore the presence of the excited dark excitons and the interband continuum makes the bright exciton visible below the band gap through level repulsions.

It should be noted that excitons without the depolarization effect may be regarded as dark excitons. In the lowest-order effective-mass scheme, optical transitions with nonzero $l$ are degenerate between the $K$ and $K^{\prime}$ points. The degeneracy can be slightly lifted due to an electronhole asymmetry or external magnetic fields. Then, new steep branches appear between pairs of lifted levels, leading to weak brightening of dark excitons. ${ }^{46,47,86}$ The oscillator strength for the lowest brightened dark exciton is proportional to $(S / L)^{2}{ }^{47}$

In the lowest-order approximation, the first excited exciton does not contribute to $\sigma_{x x}^{ \pm 1}(\omega)$ because of the presence of parity symmetry between $k>0$ and $k<0$ as shown by a dash-dotted line in Fig. 2. This symmetry is lifted when effects of trigonal warping are included, i.e., for nonzero $\beta$. In fact, the position of the band minimum or maximum for the $K$ point is shifted to

$$
k_{0}=\frac{\sqrt{3} \beta a}{4} \kappa_{\nu}^{2}(n, \varphi) \sin 3 \eta,
$$

in the absence of the Coulomb interaction and for the $K^{\prime}$ point, the signs of $\beta$ and $v$ and $\varphi$ in $\kappa_{v}(n, \varphi)$ change. This becomes different between the initial and final states in the crosspolarization geometry, destroying the parity symmetry, making the first excited exciton slightly allowed. The first excited exciton lies close to the lowest bright exciton as shown in Fig. 2. Therefore it can sometimes appear in the absorption spectrum, in particular, when its energy almost exactly coincides with that of the bright exciton, as will be shown in the next section.

\section{NUMERICAL RESULTS}

\section{A. Lowest-order effective-mass approximation}

Calculated excitation energies in the lowest-order effectivemass approximation, i.e., $\beta=p=S=0$, are shown in Fig. 3 as a function of the dielectric constant for (a) outside and (b) inside materials. For various ratios between $R_{0}$ and $R$, the energy of the lowest bright exciton is plotted by solid lines, the band gap by dotted lines, and the lowest and second excited dark excitons by dashed and dash-dotted lines, respectively.
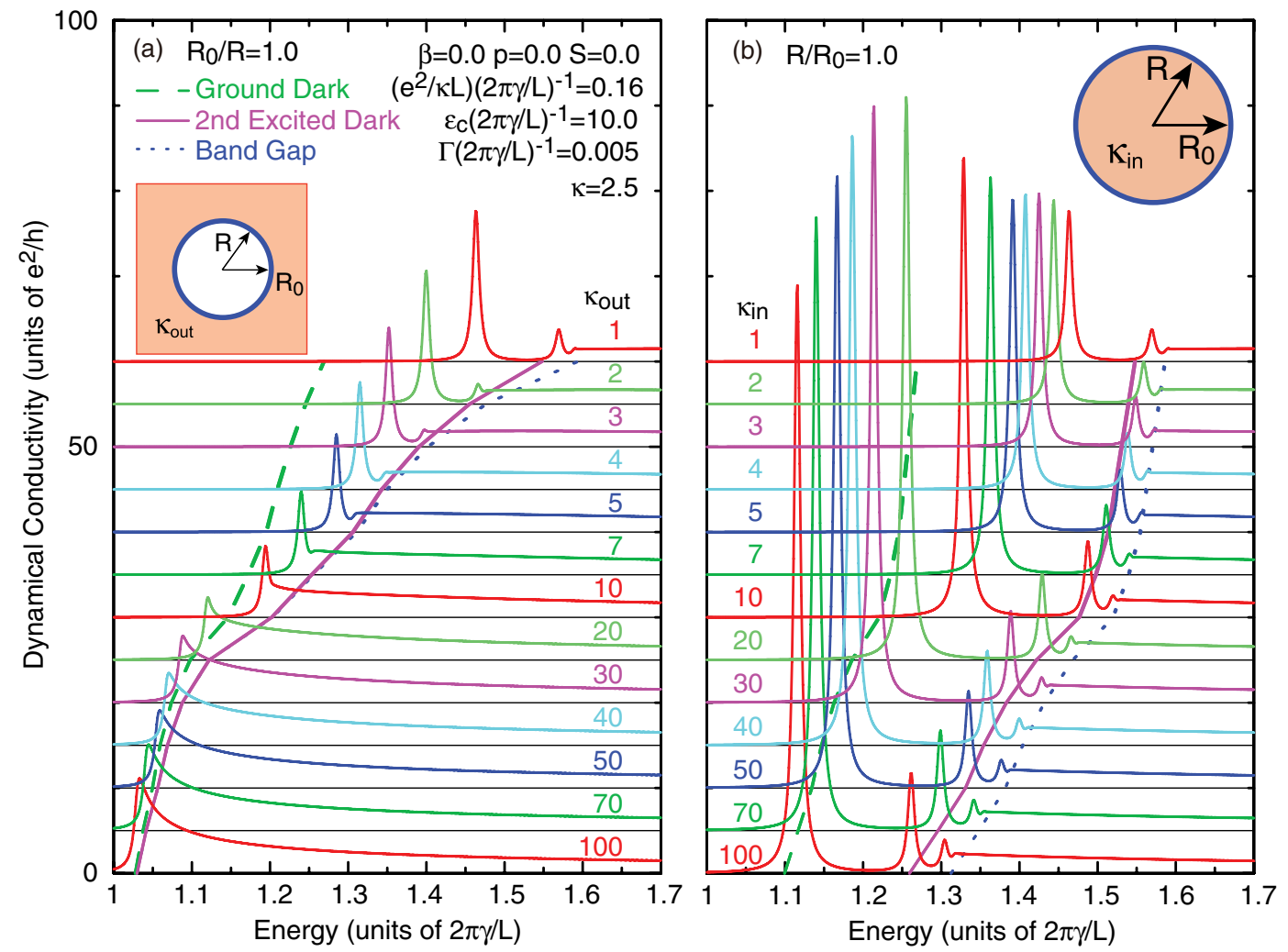

FIG. 4. (Color online) Real part of the dynamical conductivity for tubes with (a) outside and (b) inside materials for various dielectric constants in the lowest-order effective-mass approximation, i.e., $\beta=p=S=0 . R_{0}=R$. Dotted lines indicate band gaps, and dashed and solid lines the lowest and second excited dark exciton energies, respectively. Origins of vertical axes are shifted as shown by horizontal thin solid lines. Phenomenological energy broadening is introduced as $\Gamma(2 \pi \gamma / L)^{-1}=0.005$. 

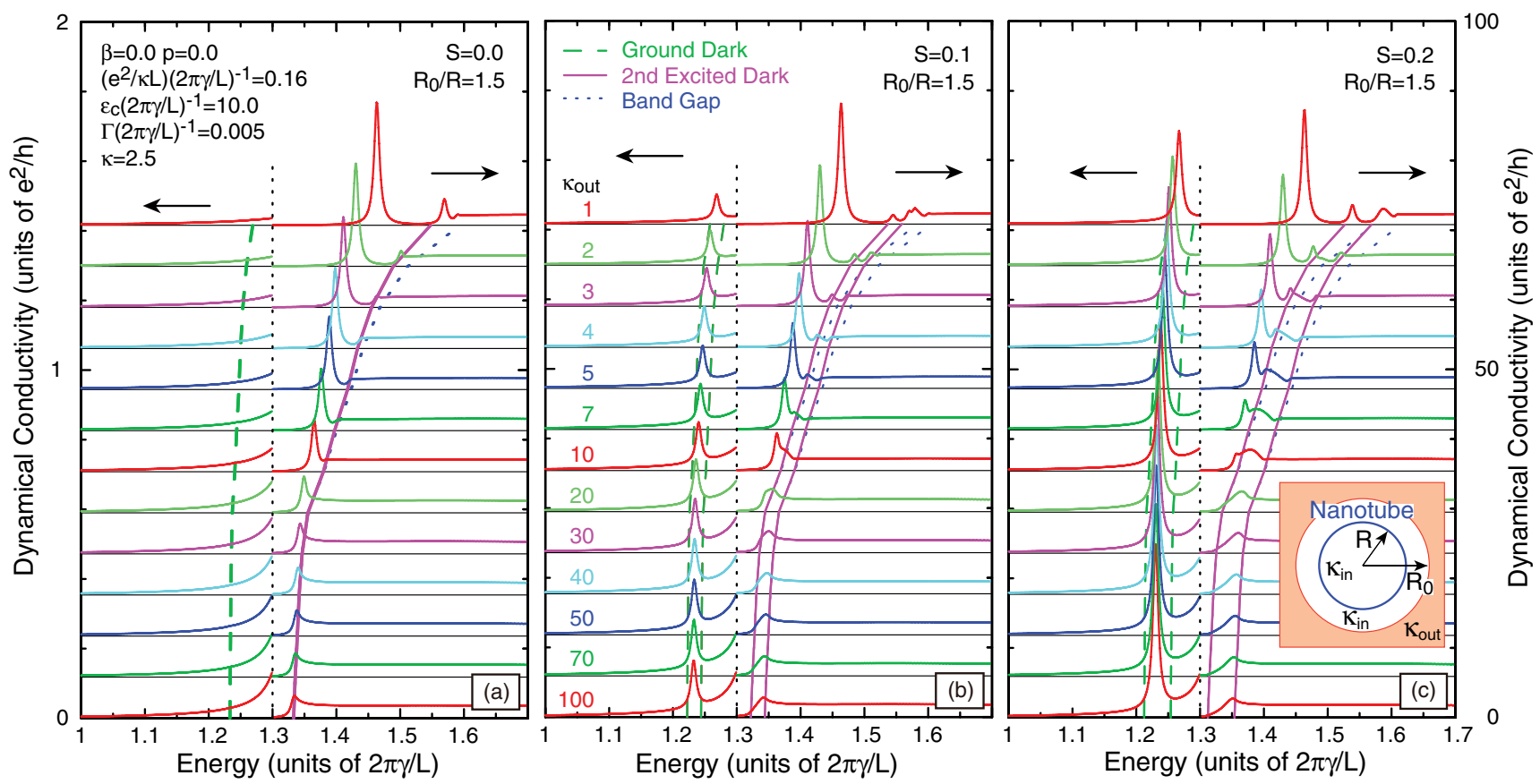

FIG. 5. (Color online) Real part of the dynamical conductivity for tubes with outside materials with $R_{0} / R=1.5$ when asymmetry between the conduction and valence bands is considered. (a) $S=0$. (b) $S=0.1$. (c) $S=0.2$. Scales of vertical axes in the energy regions lower and higher than $1.3 \times(2 \pi \gamma / L)$ are shown on the left and right sides, respectively.

The first excited dark exciton does not contribute to optical transitions due to parity symmetry as discussed in Sec. II D.

For outside materials in Fig. 3(a), all the energies decrease with the increase of $\kappa_{\text {out }}$ because of enhanced screening of the Coulomb interaction. The binding energy of the second excited dark exciton, which is the separation between the dotted and dash-dotted lines, quickly vanishes with $\kappa_{\text {out }}$ almost independently of $R_{0} / R$. This feature for excited excitons is exactly the same as that in the case of parallel polarization. ${ }^{57}$ In a similar manner, the binding energy of the bright excitons, the separation between the dotted and solid lines, is significantly reduced with $\kappa_{\text {out }}$ and disappears for sufficiently large $\kappa_{\text {out }}$. For $\kappa_{\text {out }} \sim 50$, for example, the binding energy of the lowest dark exciton, i.e., the difference between the dotted and dashed
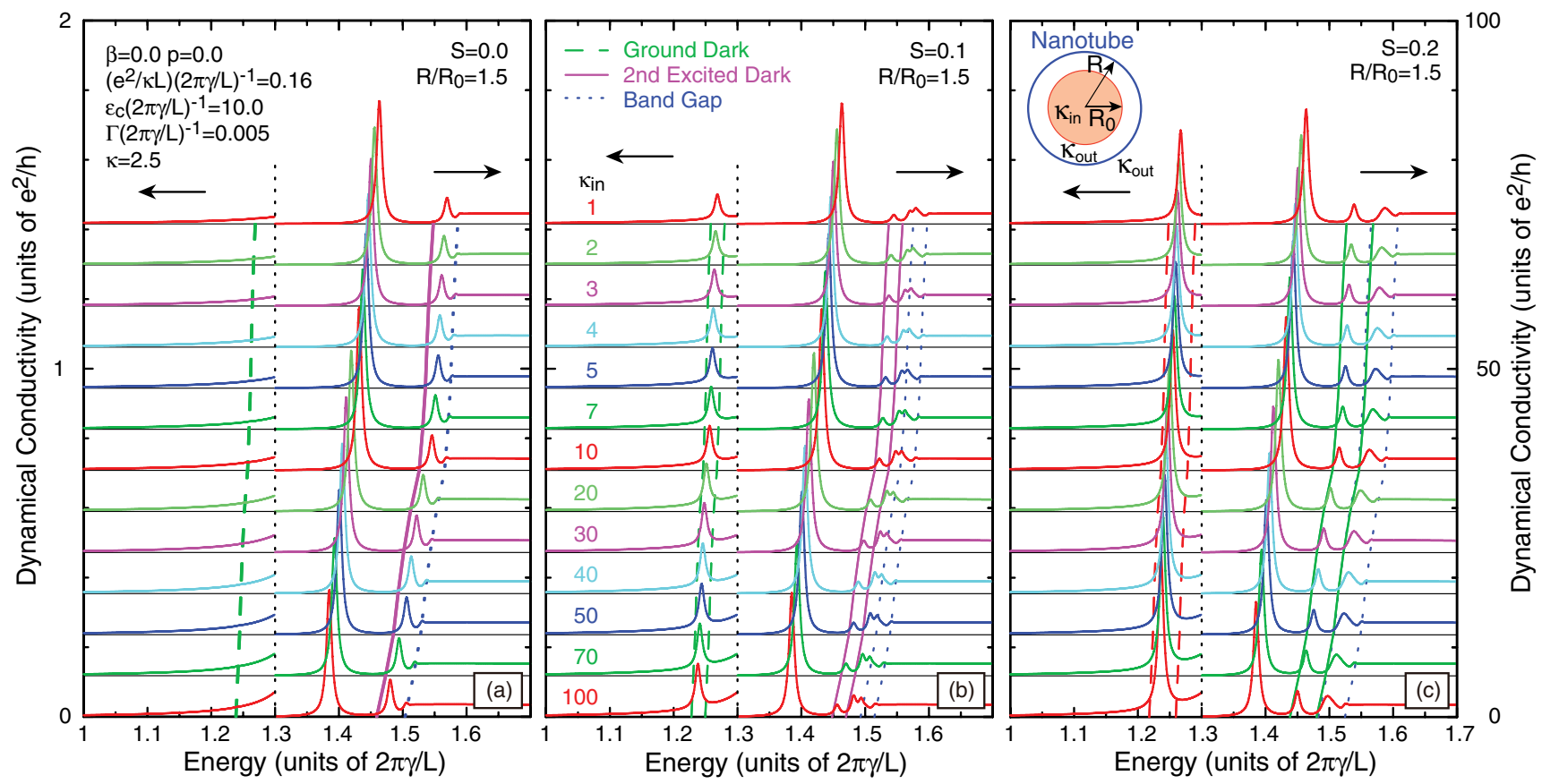

FIG. 6. (Color online) Real part of the dynamical conductivity for tubes with inside materials with $R / R_{0}=1.5$ when asymmetry between the conduction and valence bands is considered. 

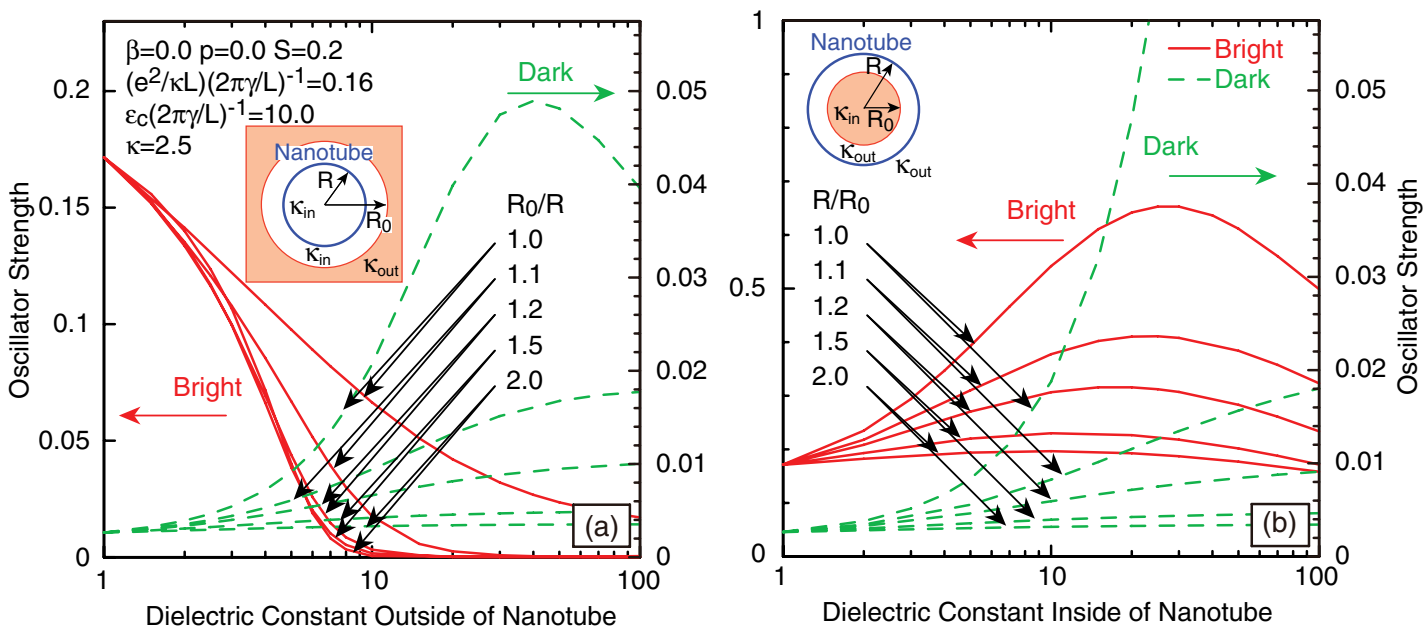

FIG. 7. (Color online) Oscillator strength for tubes with (a) outside and (b) inside materials as a function of the dielectric constant for $S=0.2$. Solid and dashed lines are for the lowest bright and the lowest brightened dark excitons, respectively.

lines, almost vanishes for $R_{0} / R=1$ and increases with the increase of $R_{0} / R$. On the other hand, for $\kappa_{\text {out }} \sim 50$ the bright exciton is already immersed into continuum states essentially independently of $R_{0} / R$.

In the case of inside materials shown in Fig. 3(b), the decrease of all the energy levels with $\kappa_{\text {in }}$ is much smaller, as has been discussed in Sec. II B. Because the second excited dark exciton survives in the whole parameter region in this figure, the lowest bright exciton remains to be dominant. In the region $\kappa_{\text {in }} \lesssim 30$ for $R / R_{0}=1$, the binding energy of the bright exciton, which is given by the difference between the dotted and solid lines, increases with the increase of the dielectric constant. This is because the depolarization effect is more effectively reduced by the inside materials in comparison with effects on electron-hole binding. This tendency is weakened with the increase of $R / R_{0}$ due to rapid decrease of environment effects on the depolarization effect.

The characteristic features of environment effects are most clearly seen in the case $R_{0}=R$. Figure 4 shows the real part of the dynamical conductivity for various values of the dielectric constant in the case of (a) outside and (b) inside materials. Band edges (dotted lines) and energies of the lowest dark excitons (dashed lines) and the second excited dark excitons (solid lines) are also drawn.

In Fig. 4(a), the intensity of main peak associated with the lowest bright exciton is rapidly reduced with the increase of
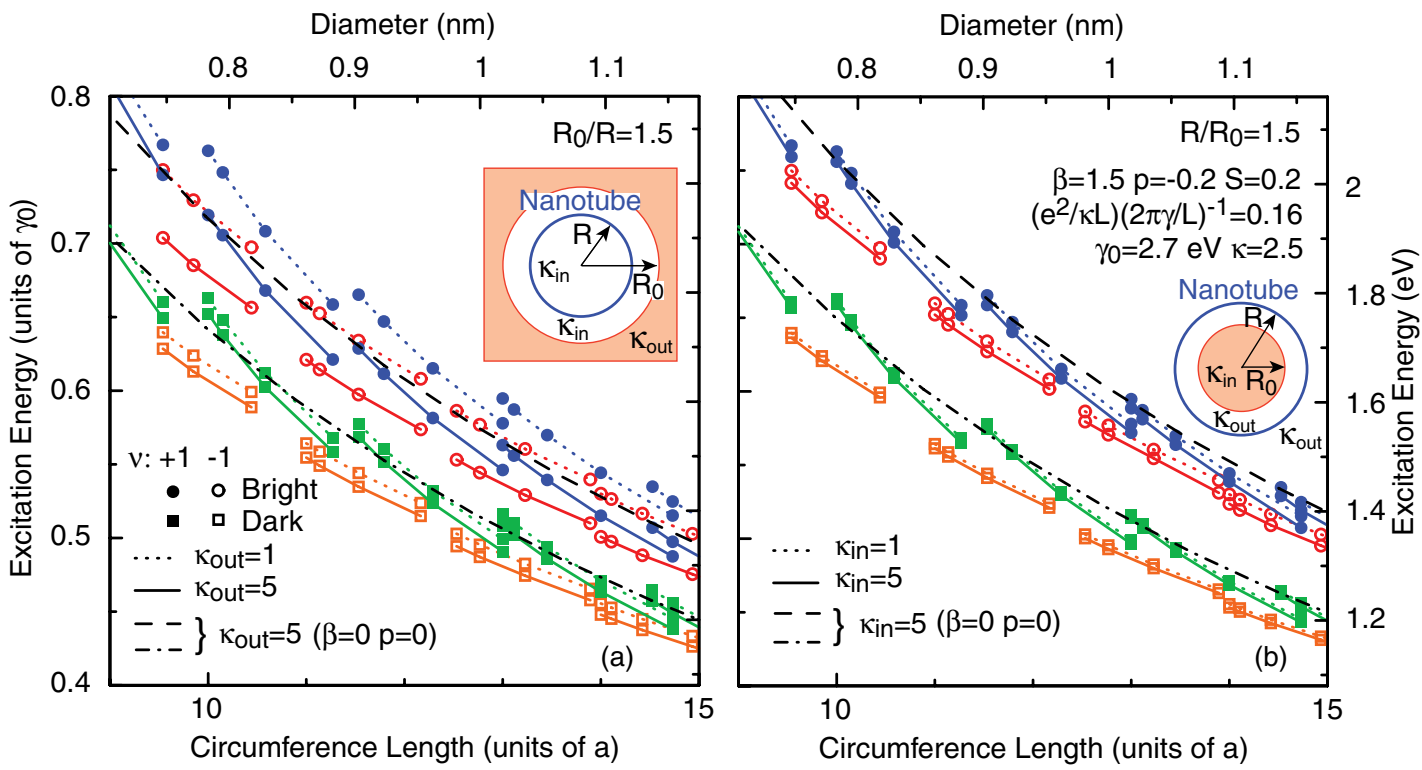

FIG. 8. (Color online) Energies of the lowest bright exciton and the brightened dark exciton as a function of circumference or diameter for (a) outside material with $R_{0} / R=1.5$ and (b) inside material with $R / R_{0}=1.5$. The parameters are $\beta=1.5, p=-0.2$, and $S=0.2$. Circles and squares denote the lowest bright and brightened dark excitons, respectively. Closed and open symbols are for $v=+1$ and -1 , respectively. Symbols with dotted and solid lines denote results in the absence and presence of dielectric materials, respectively. Corresponding results for $\beta=p=0$ and $S=0.2$ are also included by dashed and dash-dotted lines for the lowest bright and the brightened dark excitons, respectively. 
$\kappa_{\text {out }}$ and the spectrum becomes similar to one-dimensional joint density of states for sufficiently large $\kappa_{\text {out }}$. The disappearance of the exciton peaks occurs when the second excited exciton vanishes, i.e., when the solid and dotted lines merge with each other.

For inside materials in Fig. 4(b), the intensity of main peaks increases with dielectric constant $\kappa_{\text {in }}$ because of relatively larger reduction of the depolarization effect than that of the electron-hole binding as discussed above and in Sec. II B. For sufficiently large $\kappa_{\text {in }}$, the intensity saturates corresponding to the complete screening of the depolarization effect.

\section{B. Effects of electron-hole asymmetry}

For nonzero $S$, the degeneracy between transitions at the $K$ and $K^{\prime}$ points is lifted and therefore band edges and the dark excitons become different between the $K$ and $K^{\prime}$ points, giving rise to the appearance of a new peak between them (brightening of dark excitons). The calculated dynamical conductivity for $\beta=p=0$ is plotted in Fig. 5 in the case of $R_{0} / R=1.5$ for $S=0,0.1$, and 0.2 . The intensity of the brightened dark exciton grows with $S$. It becomes stronger also with the increase of the dielectric constant, corresponding to the reduced depolarization effect due to environmental screening as mentioned in Sec. II B.

In Fig. 5 for $S=0.2$, for example, two bright excitons are well resolved above the main bright exciton at $\varepsilon=$ $1.46 \times(2 \pi \gamma / L)$ for $\kappa_{\text {out }}=1$. With the increase of $\kappa_{\text {out }}$, these peaks turn into a single hump like structure lying between the split band edges. At the same time, the main bright exciton loses its intensity, merges into the hump, and then disappears completely $\left(\kappa_{\text {out }} \sim 20\right)$. With further increase of $\kappa_{\text {out }}$, the hump-like structure disappears and spectrum becomes close to interband continuum starting at the higher of two split band edges.

Figure 6 shows the similar results for inside materials with $R / R_{0}=1.5$. The brightened dark exciton appears and its intensity grows with increase of $S$. The feature of the main bright exciton remains essentially the same even for very large value of $\kappa_{\text {in }}$, which is quite different from those in the case
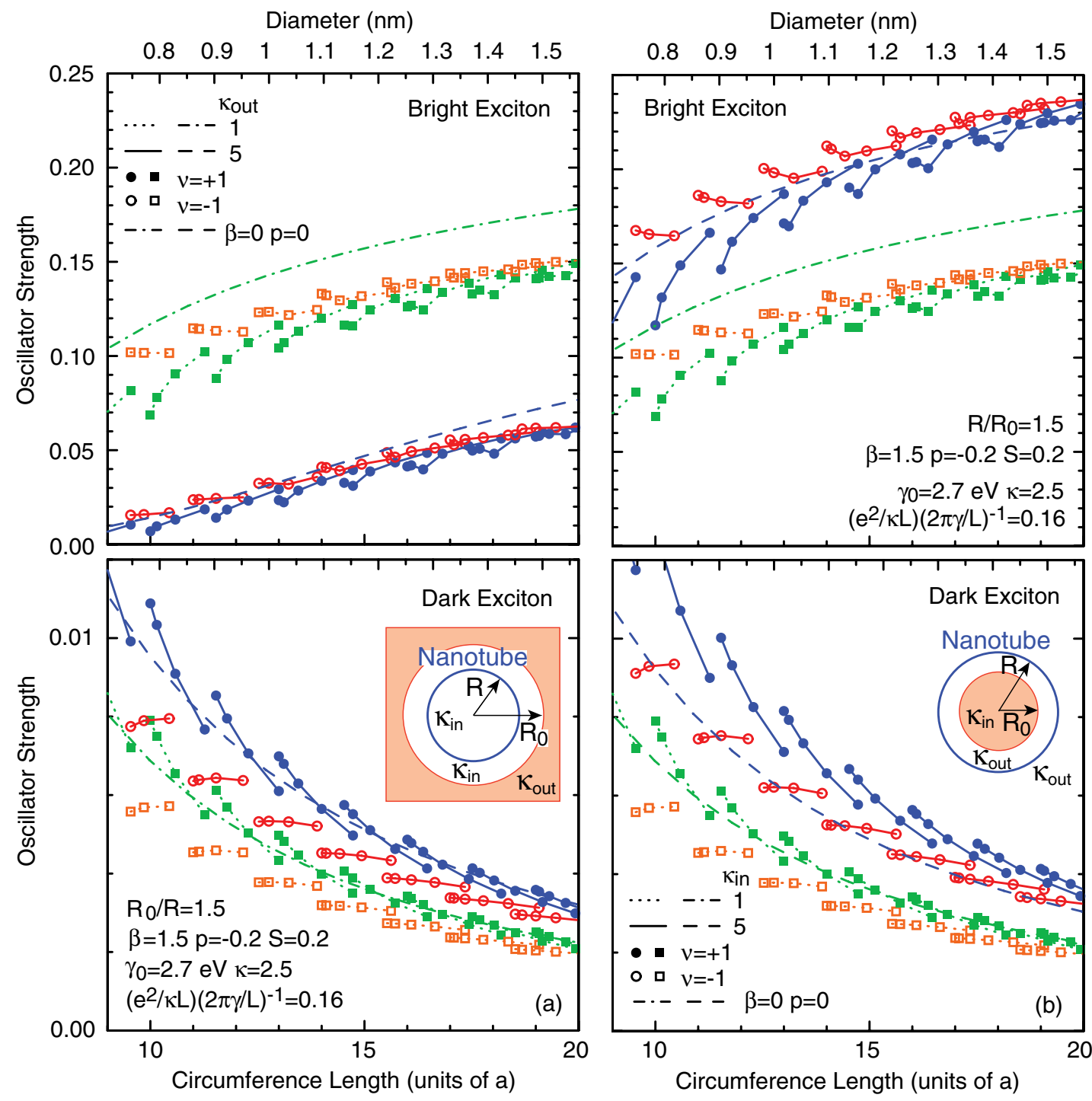

FIG. 9. (Color online) The oscillator strength for (a) inside and (b) outside materials. The top panels show that of the lowest bright exciton and the bottom panels that of the brightened dark exciton. 
of outside materials. The brightened dark exciton remains about the same position and its intensity increases with $\kappa_{\text {in }}$ corresponding to the reduction in the depolarization effect due to environmental screening.

Figure 7 shows the oscillator strength ${ }^{40}$ of the lowest bright exciton (solid lines) and the lowest brightened dark exciton (dashed lines) for (a) outside and (b) inside materials with various ratios between $R_{0}$ and $R$. The oscillator strength of the brightened dark exciton increases with the increase of the dielectric constant for both outside and inside materials. The results for the brightened dark exciton are about the same between inside and outside materials for $\kappa_{\text {out }} \lesssim 20$ and $\kappa_{\text {in }} \lesssim 20$. This is due to the symmetry of $\varepsilon_{x x, l}(\omega)$ for the outside and inside materials as has been discussed in Sec. II C, combined with the fact that the two split lowest dark excitons are dominant in determining $\sigma_{x x}^{l}(\omega)$ near the brightened dark exciton. Actually, the energy of the bright exciton is nearly independent of $S$ as long as it exhibits a distinct peak. ${ }^{47}$

\section{Family behavior}

In this section, all higher-order terms are considered. We shall choose parameters such that $\beta=1.5, p=-0.2$, and $S=0.2$ and confine ourselves to the case of $R_{0} / R=1.5$ and $\kappa_{\text {out }}=5$ for outside materials and $R / R_{0}=1.5$ and $\kappa_{\text {in }}=5$ for inside materials.

Figure 8 shows excitation energy of the lowest bright and the lowest brightened dark excitons for (a) outside and (b) inside materials as a function of the circumference length. Symbols with solid and dotted lines denote energies with and without environmental screening, respectively. In the case of outside material, environmental screening considerably reduces the energy of the bright exciton, but the effect on the brightened dark exciton is much smaller, as has previously been discussed (see Figs. 3 and 5). In the case of inside material, on the other hand, excitation energies are only slightly reduced for both bright and brightened dark excitons because of weak screening effect for $R / R_{0}>1$ (see Figs. 3 and 6). The family behavior is much weaker than in the case of parallel configuration, because of near cancellation between valence and conduction bands corresponding to optical transitions in the cross-polarization geometry. ${ }^{47}$ It is interesting to note that trigonal warping and curvature, i.e., nonzero $\beta$ and $p$, tend to reduce the exciton energies from those for $\beta=p=0$, which are shown by dashed and dash-dotted lines for the lowest bright and the brightened dark excitons, respectively.

Figure 9 shows the oscillator strength for (a) outside and (b) inside materials. The top panels show that of the lowest bright exciton and the bottom panels that of the brightened dark exciton. The oscillator strength of the bright exciton gradually increases with the increase of the circumference because of reduction of the depolarization effect arising from enhanced screening of the Coulomb interaction for larger $L{ }^{47}$ On the other hand, that of the brightened dark exciton decreases with the increase of the circumference length because it is essentially proportional to $(S / L)^{2}$ as previously shown. ${ }^{47}$

For the bright exciton, the oscillator strength is strongly reduced or enhanced by the presence of outside and inside materials, respectively. For the brightened dark exciton, on the other hand, it is enhanced in a similar manner by both inside and outside materials. These behaviors are the same as those in the lowest-order effective-mass approximation with nonzero $S$, as shown in Fig. 7. The family behavior is more prominent for the oscillator strength than the exciton energies.

Some examples of the conductivity are shown in Fig. 10 for (a) outside and (b) inside material. We choose a $(9,7)$ nanotube in (a) and a $(12,11)$ tube in (b). Dashed lines indicate the result without environmental material for reference. Arrows with
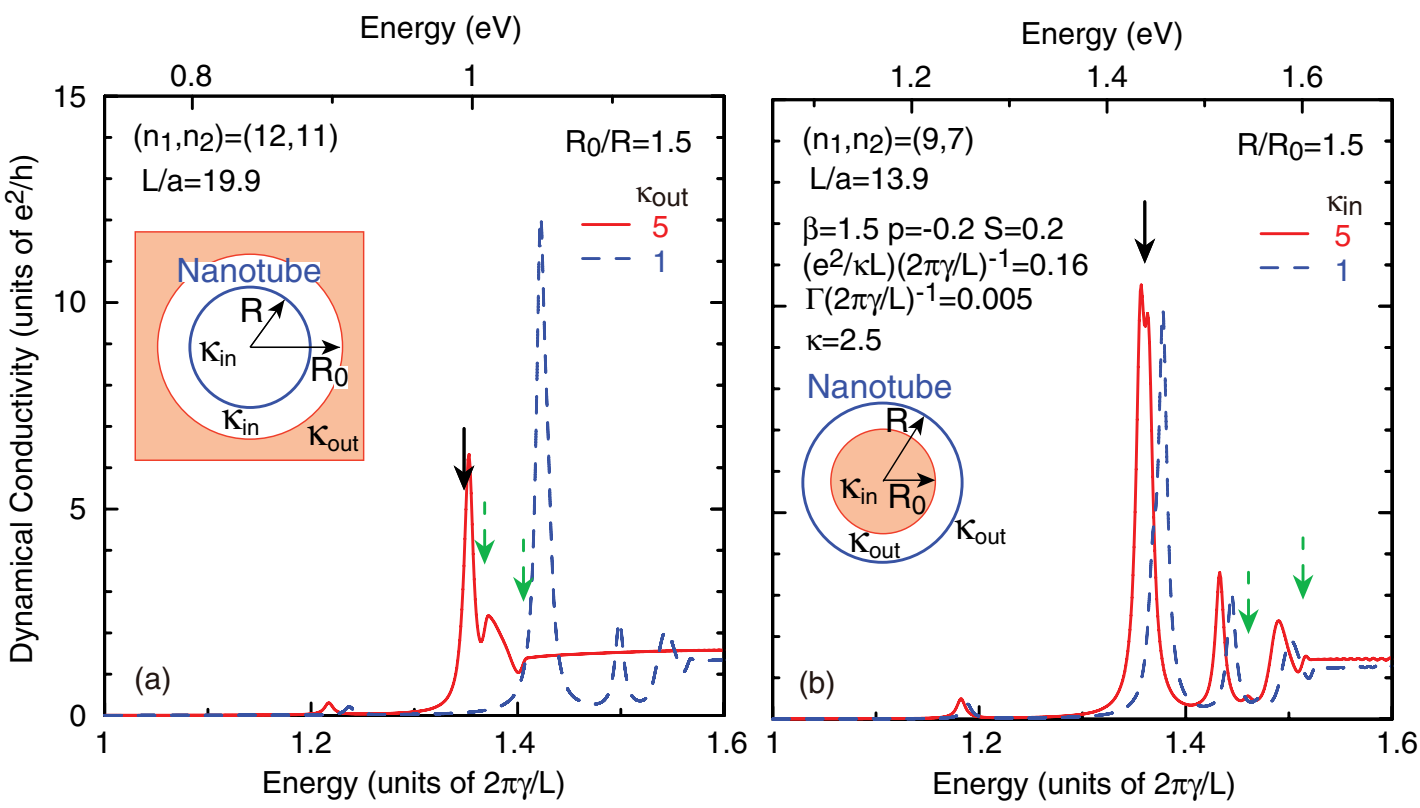

FIG. 10. (Color online) Real part of the dynamical conductivity of (a) a $(12,11)$ tube with outside material of $\kappa_{\text {out }}=5$ and $R_{0} / R=1.5$ and (b) a $(9,7)$ tube with the inside materials of $\kappa_{\text {in }}=5$ and $R / R_{0}=1.5 . \beta=1.5 . p=-0.2 . S=0.2$. Solid and dashed lines denote results with and without the materials, respectively. An arrow with a solid line denotes an energy of the first excited dark exciton and those with dashed lines band edges. 
dashed lines denote split band edges. An arrow with a solid line indicates the energy of the first excited exciton, which is forbidden by the parity symmetry in the absence of trigonal warping, i.e., $\beta=0$ and becomes slightly allowed for nonzero $\beta$, as has been discussed in Sec. II D.

For the outside material (a), the spectrum exhibits a humplike structure above the lowest bright exciton, which is the same as for $\beta=p=0$ shown in Fig. 5. The bright exciton is not affected by the presence of slightly allowed first excited exciton because their energies are sufficiently different. For the inside material (b), the spectrum is not affected by environmental screening apart from slight shift, except that a diplike structure appears at the peak of the lowest bright exciton. In this case, the first excited dark exciton happens to be degenerate with the bright exciton, causing this sharp diplike feature.

\section{DISCUSSIONS}

In this section, calculated results for outside materials with $R_{0} / R=1.5$, as a typical example, are compared with experiments. Figure 11 shows calculated energies of the lowest bright and brightened dark excitons by solid and dashed lines, respectively, for $S=0.2$ and $\beta=p=0$. As has been discussed in the previous section, the exciton energy is almost independent of $S$ and the inclusion of the other neglected corrections, $\beta$ and $p$, causes a weak family behavior and makes the energies slightly lower on average. Symbols are experiments in $\mathrm{D}_{2} \mathrm{O}$ with surfactant 44,46 and dotted lines are empirical results for tubes suspended in air. ${ }^{42}$ Crosses are measured energies of brightened dark excitons for tubes in $\mathrm{D}_{2} \mathrm{O}$ with surfactant. ${ }^{46}$ The calculated energies are semiquantitatively in good agreement with the experiments.

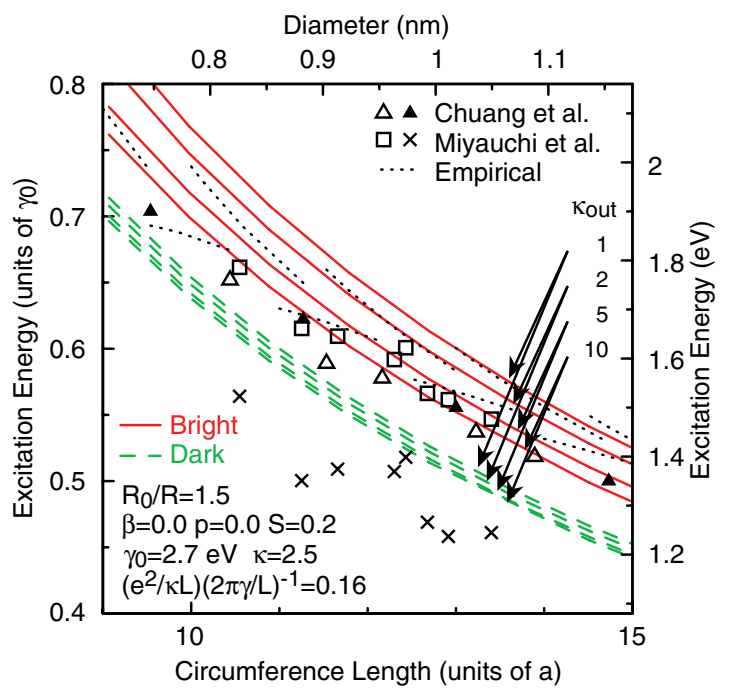

FIG. 11. (Color online) Excitation energies in the presence of outside material of $R_{0} / R=1.5$ for varying dielectric constants. $\beta=p=0 . S=0.2$. Solid and dashed lines denote those of the lowest bright and brightened dark excitons, respectively. Dotted lines denote empirical results determined experimentally, ${ }^{42}$ squares and triangles experiments for the bright exciton, ${ }^{44,46}$ and crosses those for the brightened dark exciton. ${ }^{46}$ Closed and open triangles denote $v=+1$ and -1 , respectively, and $v$ for squares is unknown.

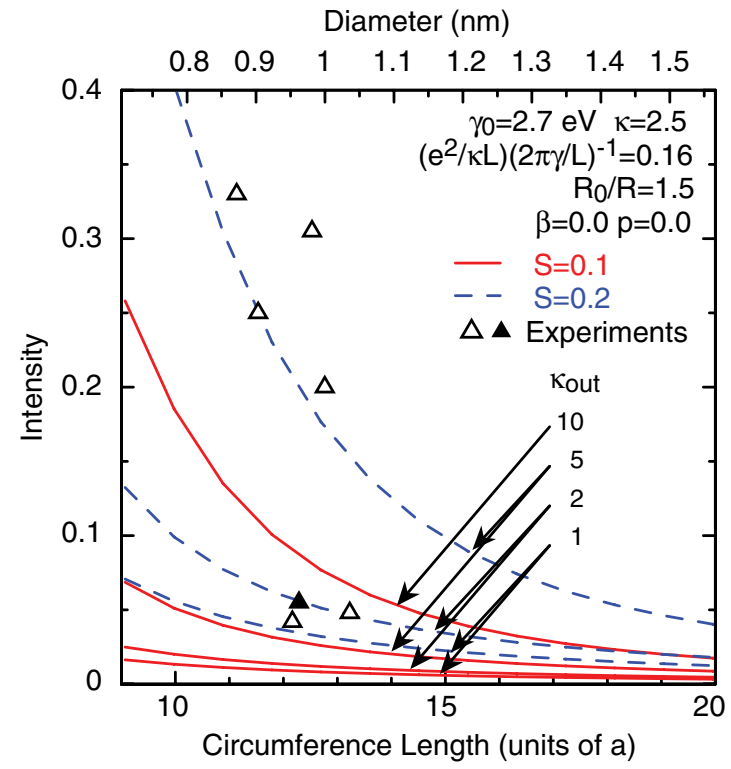

FIG. 12. (Color online) The normalized intensity of the lowest brightened dark exciton, the intensity normalized by the sum of those of the lowest bright and lowest brightened dark excitons, in the presence of outside material with $R_{0} / R=1.5$ and various dielectric constants. Solid and dashed lines are calculations with $S=0.1$ and 0.2 , respectively, where $\beta=p=0$. Triangles are experiments where closed and open symbols are $v=+1$ and -1 , respectively. ${ }^{46}$

In Fig. 12, the intensity for the lowest brightened dark excitons is compared with that of the experiments shown in Fig. $11 .^{46}$ It is normalized by the sum of those of the lowest bright and lowest brightened dark excitons. The normalized intensity $(\beta=p=0)$, denoted by solid and dashed lines for $S=$ 0.1 and 0.2 , respectively, rapidly increases with the increase of $\kappa_{\text {out }}$. The experimental results denoted by triangles are widely scattered, but may all be explained by assuming certain combinations of $\kappa_{\text {out }}$ and $S$ lying in the region $2 \lesssim \kappa_{\text {out }} \lesssim 10$ and $0.1 \lesssim S \lesssim 0.2$. Detailed information about the environment is necessary for making more precise comparison possible, including the determination of asymmetry parameter $S$.

\section{SUMMARY AND CONCLUSION}

Effects of dielectric environments on cross-polarized excitons have been theoretically studied in the effective-mass approximation. When a nanotube is surrounded by outside material, the lowest bright exciton is drastically weakened and merges into the interband continuum with the increase of the dielectric constant of the environment material. This is because excited excitons limiting the depolarization shift easily disappear by environmental screening. When a nanotube contains inside material, on the other hand, the lowest bright exciton is enhanced because depolarization field is more effectively screened than the Coulomb interaction causing exciton formation. The brightened dark exciton due to the electron-hole asymmetry becomes more and more prominent with the increase of the environmental dielectric constant both for inside and outside materials. Consequently, the relative intensity of the brightened dark exciton normalized 
by the bright exciton is strongly enhanced by environmental screening, in particular, for outside materials.

\section{ACKNOWLEDGMENTS}

We thank H. Suzuura and Y. Tomio for fruitful discussions. This work was supported in part by a Global Center of Excellence Program at Tokyo Tech "Nanoscience and Quantum Physics," Grants-in-Aid for Scientific Research, and Grant-in-Aid for Scientific Research on Priority Area "Carbon Nanotube Nanoelectronics" from the Ministry of Education, Culture, Sports, Science and Technology, Japan. The numerical calculations were partly performed at Iwate University Super Computing and Information Sciences Center.
${ }^{1}$ V. C. Moore, M. S. Strano, E. H. Haroz, R. H. Hauge, R. E. Smalley, J. Schmidt, and Y. Talmon, Nano Lett. 3, 1379 (2003).

${ }^{2}$ S. G. Chou, H. B. Ribeiro, E. B. Barros, A. P. Santos, D. Nezich, G. G. Samsonidze, C. Fantini, M. A. Pimenta, A. Jorio, F. Plentz Filho, M. S. Dresselhaus, G. Dresselhaus, R. Saito, M. Zheng, G. B. Onoa, E. D. Semke, A. K. Swan, M. S. Ünlü, and B. B. Goldberg, Chem. Phys. Lett. 397, 296 (2004).

${ }^{3}$ C. Fantini, A. Jorio, M. Souza, M. S. Strano, M. S. Dresselhaus, and M. A. Pimenta, Phys. Rev. Lett. 93, 147406 (2004).

${ }^{4}$ J. Lefebvre, J. M. Fraser, Y. Homma, and P. Finnie, Appl. Phys. A 78, 1107 (2004).

${ }^{5}$ T. Hertel, A. Hagen, V. Talalaev, K. Arnold, F. Hennrich, M. Kappes, S. Rosenthal, J. McBride, H. Ulbricht, and E. Flahaut, Nano Lett. 5, 511 (2005).

${ }^{6}$ D. A. Tsyboulski, S. M. Bachilo, and R. B. Weisman, Nano Lett. 5, 975 (2005).

${ }^{7}$ T. Okazaki, T. Saito, K. Matsuura, S. Ohshima, M. Yumura, and S. Iijima, Nano Lett. 5, 2618 (2005).

${ }^{8}$ Y. Ohno, S. Iwasaki, Y. Murakami, S. Kishimoto, S. Maruyama, and T. Mizutani, Phys. Rev. B 73, 235427 (2006).

${ }^{9}$ Y. Ohno, S. Iwasaki, Y. Murakami, S. Kishimoto, S. Maruyama, and T. Mizutani, Phys. Stat. Sol. B 244, 4002 (2007).

${ }^{10}$ O. Kiowski, S. Lebedkin, F. Hennrich, S. Malik, H. Rosner, K. Arnold, C. Surgers, and M. M. Kappes, Phys. Rev. B 75, 075421 (2007).

${ }^{11}$ A. G. Walsh, A. N. Vamivakas, Y. Yin, S. B. Cronin, M. S. Ünlü, B. B. Goldberg, and A. K. Swan, Nano Lett. 7, 1485 (2007).

${ }^{12}$ P. T. Araujo and A. Jorio, Phys. Stat. Sol. B 245, 2201 (2008).

${ }^{13}$ R. K. Wang, W.-C. Chen, D. K. Campos, and K. J. Ziegler, J. Am. Chem. Soc. 130, 16330 (2008).

${ }^{14}$ J. G. Duque, M. Pasquali, L. Cognet, and B. Lounis, ACS Nano 3, 2153 (2009).

${ }^{15}$ C. A. Silvera-Batista, R. K. Wang, P. Weinberg, and K. J. Ziegler, Phys. Chem. Chem. Phys. 12, 6990 (2010).

${ }^{16}$ L. Lüer, S. Hoseinkhani, M. Meneghetti, and G. Lanzani, Phys. Rev. B 81, 155411 (2010).

${ }^{17}$ R. J. Elliot and R. Loudon, J. Phys. Chem. Solids 8, 382 (1959).

${ }^{18}$ R. Loudon, Am. J. Phys. 27, 649 (1959).

${ }^{19}$ T. Ogawa and T. Takagahara, Phys. Rev. B 43, 14325 (1991).

${ }^{20}$ T. Ando, J. Phys. Soc. Jpn. 66, 1066 (1997).

${ }^{21}$ T. Ando, J. Phys. Soc. Jpn. 73, 3351 (2004).

${ }^{22}$ V. Perebeinos, J. Tersoff, and Ph. Avouris, Phys. Rev. Lett. 92, 257402 (2004).

${ }^{23}$ C. D. Spataru, S. Ismail-Beigi, L. X. Benedict, and S. G. Louie, Phys. Rev. Lett. 92, 077402 (2004).

${ }^{24}$ C. D. Spataru, S. Ismail-Beigi, R. B. Capaz, and S. G. Louie, Phys. Rev. Lett. 95, 247402 (2005).

${ }^{25}$ C. L. Kane and E. J. Mele, Phys. Rev. Lett. 93, 197402 (2004).
${ }^{26}$ E. Chang, G. Bussi, A. Ruini, and E. Molinari, Phys. Rev. Lett. 92, 196401 (2004)

${ }^{27}$ E. Chang, G. Bussi, A. Ruini, and E. Molinari, Phys. Rev. B 72, 195423 (2005).

${ }^{28}$ H. Zhao and S. Mazumdar, Phys. Rev. Lett. 93, 157402 (2004).

${ }^{29}$ H. Zhao, S. Mazumdar, C.-X. Sheng, M. Tong, and Z. V. Vardeny, Phys. Rev. B 73, 075403 (2006).

${ }^{30}$ Z. Wang, H. Zhao, and S. Mazumdar, Phys. Rev. B 74, 195406 (2006).

${ }^{31}$ M. Ichida, S. Mizuno, Y. Tani, Y. Saito, and A. Nakamura, J. Phys. Soc. Jpn. 68, 3131 (1999).

${ }^{32}$ M. Ichida, S. Mizuno, Y. Saito, H. Kataura, Y. Achiba, and A. Nakamura, Phys. Rev. B 65, 241407 (2002).

${ }^{33}$ M. J. O'Connell, S. M. Bachilo, C. B. Huffman, V. C. Moore, M. S. Strano, E. H. Haroz, K. L. Rialon, P. J. Boul, W. H. Noon, C. Kittrell, J. Ma, R. H. Hauge, R. B. Weisman, and R. E. Smalley, Science 297, 593 (2002).

${ }^{34}$ S. M. Bachilo, M. S. Strano, C. Kittrell, R. H. Hauge, R. E. Smalley, and R. B. Weisman, Science 298, 2361 (2002).

${ }^{35}$ F. Wang, G. Dukovic, L. E. Brus, and T. F. Heinz, Science 308, 838 (2005).

${ }^{36}$ J. Maultzsch, R. Pomraenke, S. Reich, E. Chang, D. Prezzi, A. Ruini, E. Molinari, M. S. Strano, C. Thomsen, and C. Lienau, Phys. Rev. B 72, 241402 (2005).

${ }^{37}$ G. Dukovic, F. Wang, D. Song, M. Y. Sfeir, T. F. Heinz, and L. E. Brus, Nano Lett. 5, 2314 (2005).

${ }^{38}$ H. Ajiki and T. Ando, Physica B 201, 349 (1994).

${ }^{39}$ H. Ajiki and T. Ando, Jpn. J. Appl. Phys. Suppl. 34-1, 107 (1995).

${ }^{40}$ S. Uryu and T. Ando, Phys. Rev. B 74, 155411 (2006).

${ }^{41}$ Y. Miyauchi, M. Oba, and S. Maruyama, Phys. Rev. B 74, 205440 (2006).

${ }^{42}$ J. Lefebvre and P. Finnie, Phys. Rev. Lett. 98, 167406 (2007).

${ }^{43}$ Z.-D. Wang, H.-B. Zhao, and S. Mazumdar, Phys. Rev. B 76, 115431 (2007).

${ }^{44}$ K.-C. Chuang, A. Nish, J.-Y. Hwang, G. W. Evans, and R. J. Nicholas, Phys. Rev. B 78, 085411 (2008).

${ }^{45}$ H. Ajiki, J. Phys.: Conf. Ser. 210, 012049 (2010).

${ }^{46}$ Y. Miyauchi, H. Ajiki, and S. Maruyama, Phys. Rev. B 81, 121415 (2010).

${ }^{47}$ S. Uryu and T. Ando, Phys. Rev. B 83, 085404 (2011).

${ }^{48}$ P. H. Tan, A. G. Rozhin, T. Hasan, P. Hu, V. Scardaci, W. I. Milne, and A. C. Ferrari, Phys. Rev. Lett. 99, 137402 (2007).

${ }^{49}$ H. Hirori, K. Matsuda, and Y. Kanemitsu, Phys. Rev. B 78, 113409 (2008).

${ }^{50}$ A. Nakamura, N. Hikosaka, S. Imamura, Y. Takahashi, H. Ago, and H. Kishida, J. Lumin. 129, 1722 (2009).

${ }^{51}$ J. Lefebvre, Y. Homma, and P. Finnie, Phys. Rev. Lett. 90, 217401 (2003). 
${ }^{52}$ B. W. Smith, M. Monthioux, and D. E. Luzzi, Nature (London) 396, 323 (1998).

${ }^{53}$ Y. Maniwa, H. Kataura, M. Abe, A. Udaka, S. Suzuki, Y. Achiba, H. Kira, K. Matsuda, H. Kadowaki, and Y. Okabe, J. Chem. Phys. Lett. 401, 534 (2005).

${ }^{54}$ J. Jiang, R. Saito, G. G. Samsonidze, A. Jorio, S. G. Chou, G. Dresselhaus, and M. S. Dresselhaus, Phys. Rev. B 75, 035407 (2007).

${ }^{55}$ K. Sato, R. Saito, J. Jiang, G. Dresselhaus, and M. S. Dresselhaus, Phys. Rev. B 76, 195446 (2007).

${ }^{56}$ P. T. Araujo, A. Jorio, M. S. Dresselhaus, K. Sato, and R. Saito, Phys. Rev. Lett. 103, 146802 (2009).

${ }^{57}$ T. Ando, J. Phys. Soc. Jpn. 79, 024706 (2010).

${ }^{58}$ V. M. Adamyan, O. A. Smyrnov, and S. V. Tishchenko, J. Phys.: Conf. Ser. 129, 012012 (2008).

${ }^{59}$ Y. Miyauchi, R. Saito, K. Sato, Y. Ohno, S. Iwasaki, T. Mizutani, J. Jiang, and S. Maruyama, Chem. Phys. Lett. 442, 394 (2007).

${ }^{60}$ J.-H. Choi and M. S. Strano, Appl. Phys. Lett. 90, 223114 (2007).

${ }^{61}$ A. R. T. Nugraha, R. Saito, K. Sato, P. T. Araujo, A. Jorio, and M. S. Dresselhaus, Appl. Phys. Lett. 97, 091905 (2010).

${ }^{62}$ M. Rohlfing, Phys. Rev. Lett. 108, 087402 (2012).

${ }^{63}$ Y. Tomio, H. Suzuura, and T. Ando, Phys. Rev. B 85, 085411 (2012).

${ }^{64}$ T. Ando, J. Phys. Soc. Jpn. 74, 777 (2005).

${ }^{65}$ H. Ajiki and T. Ando, J. Phys. Soc. Jpn. 62, 1255 (1993).

${ }^{66}$ J. C. Slonczewski and P. R. Weiss, Phys. Rev. 109, 272 (1958).
${ }^{67}$ H. Ajiki and T. Ando, J. Phys. Soc. Jpn. 65, 505 (1996).

${ }^{68}$ C. L. Kane and E. J. Mele, Phys. Rev. Lett. 78, 1932 (1997).

${ }^{69}$ T. Ando, J. Phys. Soc. Jpn. 69, 1757 (2000).

${ }^{70}$ C. L. Kane, E. J. Mele, R. S. Lee, J. E. Fischer, P. Petit, H. Dai, A. Thess, R. E. Smalley, A. R. M. Verschueren, S. J. Tans, and C. Dekker, Europhys. Lett. 41, 683 (1998).

${ }^{71}$ H. Suzuura and T. Ando, Physica E 6, 864 (2000).

${ }^{72}$ H. Suzuura and T. Ando, Mol. Cryst. Liq. Cryst. 340, 731 (2000).

${ }^{73}$ H. Suzuura and T. Ando, Phys. Rev. B 65, 235412 (2002).

${ }^{74}$ S. Uryu, H. Ajiki, and T. Ando, Phys. Rev. B 78, 115414 (2008).

${ }^{75}$ T. Ando, J. Phys. Soc. Jpn. 78, 104703 (2009).

${ }^{76}$ R. Saito, G. Dresselhaus, and M. S. Dresselhaus, Physical Properties of Carbon Nanotubes, (Imperial College Press, London, 1998).

${ }^{77}$ T. Ando, J. Phys. Soc. Jpn. 75, 024707 (2006).

${ }^{78}$ H. Sakai, H. Suzuura, and T. Ando, J. Phys. Soc. Jpn. 72, 1698 (2003).

${ }^{79}$ H. Sakai, H. Suzuura, and T. Ando, Physica E 22, 704 (2004).

${ }^{80}$ M. Rohlfing and S. G. Louie, Phys. Rev. Lett. 80, 3320 (1998).

${ }^{81}$ M. Rohlfing and S. G. Louie, Phys. Rev. B 62, 4927 (2000).

${ }^{82}$ H. Ajiki, T. Iida, T. Ishikawa, S. Uryu, and H. Ishihara, Phys. Rev. B 80, 115437 (2009).

${ }^{83}$ M. Grüning, A. Marini, and X. Gonze, Nano Lett. 9, 2820 (2009).

${ }^{84}$ S. Uryu and T. Ando, J. Phys.: Conf. Ser. 302, 012004 (2011).

${ }^{85}$ R. Kubo, J. Phys. Soc. Jpn. 12, 570 (1957).

${ }^{86}$ S. Uryu and T. Ando, Phys. Rev. B 76, 115420 (2007). 